\title{
A systematic review of statistical models and outcomes of predicting fatal and serious injury crashes from driver crash and offense history data
}



\begin{abstract}
Background: Expenditure on driver-related behavioral interventions and road use policy is often justified by their impact on the frequency of fatal and serious injury crashes. Given the rarity of fatal and serious injury crashes, offense history, and crash history of drivers are sometimes used as an alternative measure of the impact of interventions and changes to policy. The primary purpose of this systematic review was to assess the rigor of statistical modeling used to predict fatal and serious crashes from offense history and crash history using a purpose-made quality assessment tool. A secondary purpose was to explore study outcomes.

Methods: Only studies that used observational data and presented a statistical model of crash prediction from offense history or crash history were included. A quality assessment tool was developed for the systematic evaluation of statistical quality indicators across studies. The search was conducted in June 2019.

Results: One thousand one hundred and five unique records were identified, 252 full texts were screened for inclusion, resulting in 20 studies being included in the review. The results indicate substantial and important limitations in the modeling methods used. Most studies demonstrated poor statistical rigor ranging from low to middle quality. There was a lack of confidence in published findings due to poor variable selection, poor adherence to statistical assumptions relating to multicollinearity, and lack of validation using new data.

Conclusions: It was concluded that future research should consider machine learning to overcome correlations in the data, use rigorous vetting procedures to identify predictor variables, and validate statistical models using new data to improve utility and generalizability of models.
\end{abstract}

Systematic review registration: PROSPERO CRD42019137081

Keywords: Systematic review, Quality assessment tool, Crash, Traffic, Offense, Statistics, Statistical modeling, Driver offenses, Crash history

\footnotetext{
* Correspondence: rslikboer@swin.edu.au

Centre for Mental Health, Faculty of Health Arts and Design, Swinburne University of Technology, PO Box 218, Mail H31, John St, Hawthorn, Victoria 3122, Australia
}

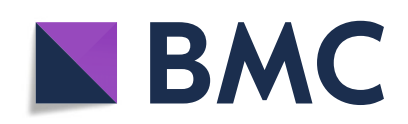

(c) The Author(s). 2020 Open Access This article is licensed under a Creative Commons Attribution 4.0 International License, which permits use, sharing, adaptation, distribution and reproduction in any medium or format, as long as you give appropriate credit to the original author(s) and the source, provide a link to the Creative Commons licence, and indicate if changes were made. The images or other third party material in this article are included in the article's Creative Commons licence, unless indicated otherwise in a credit line to the material. If material is not included in the article's Creative Commons licence and your intended use is not permitted by statutory regulation or exceeds the permitted use, you will need to obtain permission directly from the copyright holder. To view a copy of this licence, visit http://creativecommons.org/licenses/by/4.0/ The Creative Commons Public Domain Dedication waiver (http://creativecommons.org/publicdomain/zero/1.0/) applies to the data made available in this article, unless otherwise stated in a credit line to the data. 


\section{Background}

Expenditure on driver-related behavioral interventions and road use policy is often justified by their impact on the frequency of fatal and serious injury (FSI) crashes. Fatal and serious injury includes death and injuries that endanger human life, including fetuses. Injury can be acute, cumulative, and protracted [1]. Due to the increased rarity of FSI crashes, because of factors such as improved vehicle design and road infrastructure, reliably evaluating the short- and medium-term impact of interventions on FSI crashes is challenging. On the other hand, traffic offenses (e.g., speeding and disobeying traffic lights) are much more frequent than FSI crashes [2, 3]. This has led to organizations using offending patterns as a proxy measure to evaluate the effectiveness of new interventions and policies targeting the reduction of FSI crashes.

Predictive models of FSI crashes can be complex and include variables from multiple domains. Environmental factors, road conditions, legal factors, licensing factors, and driver characteristics have all been found to contribute to FSI crash involvement [4-12]. Offense history (i.e., the number of traffic infringements a driver has incurred) and crash history (i.e., the number of crashes a driver has been involved in) have also frequently been found to be useful predictors of future FSI crashes [13-15]. Offense histories that include repeated violations over time, such as exceeding the speed limit and failure to obey road signs, have been found to increase crash risk. The increase in crash risk is particularly high when repeated violations lead to license suspension or revocation. Involvement in multiple crashes over time is a stronger predictor of future crash involvement than traffic violations $[13,14,16]$. Indeed, even particularly risky offenses do not appear to always increase the risk of FSI crashes. For example, Leal and Watson [17] reported that for those who engage in illegal street racing only $3.7 \%$ of offenses result in crashes, none of them being fatal.

A handful of reviews have investigated the modeling of FSI crashes [18-22]. A common critique in these reviews concerns the suitability of the traditional statistical techniques that have been applied to this data [22]. Considering the wide-ranging implications for developing and employing a statistical model to help inform decisions around policy and funding, it is vital that models are developed using rigorous and suitable methods, producing models that can be understood by non-technical audiences. Our review emphasizes the statistical approaches and methodologies applied to modelling FSI crash data. However, we were unable to find an existing quality assessment tool that met this need and have therefore developed our own.

Further, while statistical heterogeneity between primary studies is regularly noted as a limitation in systematic reviews and meta-analyses, detail such as the rigor and suitability of the modeling methods, as well as the ease of model interpretation is rarely discussed [9, 23-27]. In this review, we focus on an area of importance to policy makers. We focus exclusively on how offense history and crash history predict future FSI crashes and how such associations are best modeled. No previous reviews have had this focus and a new quality assessment tool has been developed specifically for this review because nothing suitable could be found.

\section{Aims}

The overall research question was to assess the state of evidence for the prediction of FSI crashes from offense history and/or crash history by completing a systematic review of published literature and grey literature. Based on the failure to identify a systematic review focused on our research question and failure to identify a statistical quality assessment tool, two specific aims were formed. The first aim was to determine the type and quality of statistical analyses applied to the prediction of FSI crashes from driver offense and crash history. The quality of the papers included in the review was assessed based on the reporting of statistical assumptions, the reporting of statistical results, and the reporting of considerations specific to the statistical methods used. The second aim was to summarize the evidence and outcomes of studies that include offense history and crash history as predictors of FSI crashes.

\section{Methods \\ Protocol and registration}

The Preferred Reporting Items for Systematic Reviews and Meta-Analyses (PRISMA) were followed for the review [28]. The protocol was registered with the International Prospective Register of Systematic Reviews (PROSPERO), registration number: CRD42019137081.

\section{Inclusion and exclusion criteria Individuals and outcome of interest}

There were no restrictions on the type of individuals (i.e., drivers) included in the review. Efficacy and outcomes of interventions were not the subject of this review. Rather, we were interested in how the prediction of FSI road crashes has been modeled when described by driver offense and crash history. FSI crashes are the outcome of interest. A fatality is defined as a death that results from a crash, while serious injury resulting from a crash is defined as long-term impairment or loss of body function, permanent serious disfigurement, severe long-term mental, or severe long-term behavioral disturbance or disorder, or loss of a fetus [1]. Studies that examined crashes that did not result in fatalities or serious injury were not included in this review. 


\section{Data}

Only models based on observational data were included. Data derived from laboratory tests, simulated data, selfreport measures, and driving simulations were excluded. Studies that presented models built on qualitative data, mixed qualitative and quantitative data, and simulated data were excluded. Instantaneous traffic data used in real-time traffic crash prediction were excluded because these models typically apply only to a small section of the road.

\section{Model specifications}

To be included in the review studies must have presented a quantitative model predicting FSI crashes for individual drivers. That is, FSI crashes must be the outcome variable. A model was excluded from the review if the dependent variable included only minor crashes, such as when those involved in the accident received cuts and bruises and the vehicle had minor/repairable damage. When the dependent variable included a combination of serious and minor crashes, the model was excluded. Models had to describe crash risk based on driver offense and/or crash history. Models using longitudinal data were included. Models that considered only summarized country or state level offense and crash data, rather than individual driver data as the unit of interest, were excluded.

\section{Study design}

A study or report was included if it was a primary study that presented a quantitative model predicting FSI crashes. Study designs that were excluded included those which reported only qualitative data, reviews, metaanalyses, case studies, and any study that did not present a model for crash prediction.

\section{Publication date}

Studies and reports published prior to 1984 were excluded because data without a digital format was rare prior to this date, making statistical analysis difficult.

\section{Search strategy}

Four academic databases were systematically searched: Australian Transport Index: ATRI database (via informat); Transportation Research International Documentation (ITRD) database; Scopus; and Web of Science. Search terms were chosen to identify papers that included statistical models, serious crashes, and variables related to the driver (Appendix S1). For example for Scopus we used the following search string: (predict* OR model) AND ("serious crash" OR "serious accident" OR "serious collision" OR "fatal accident" OR "fatal crash" OR "fatal collision" OR "road deaths" OR "road fatal"”
OR "traffic fatal" " OR "collision fatal"* OR "accident fatal*") AND (driver).

Grey literature was also searched for government reports of crash prediction. The grey literature search was focused on, but not limited to, high-income countries that have low road traffic fatality rates, as indicated by the World Health Organization [29] (i.e., < 19.9 deaths per 100,000 population per annum). This was to increase the chance of identifying relevant reports in relation to the time spent searching. The search for grey literature utilized Google and Google Scholar to identify relevant international organizations, their websites, and information repositories. A total of 58 official government and statistic websites were searched from 38 countries (Appendix S2).

A forward and backward search was conducted on the articles that met the inclusion criteria. Forward searching involved identifying articles that cited the included study. Forward searching was conducted in Scopus. The backward search consisted of screening the references of the included studies (Appendix S3).

\section{Selection of studies}

Initial search records were recorded in a Word document. Every article title and the first author from each article were systematically entered into the "find" function to identify and remove duplicates. The first author conducted all searches, eliminated duplicates, screened titles, and abstracts against the inclusion/exclusion criteria, and produced a list for full-text screening. If the full text was not available, the record was excluded from the review at the title/abstract screen stage. An auditing trail was created using an inclusion/exclusion checklist for the full-text screen in Excel, in which reasons for exclusion were recorded. Two authors screened studies at the full-text stage in Excel; discrepancies were resolved by a third author.

\section{Quality assessment tool}

Statistical models were assessed in three areas using a purpose-made statistical quality assessment tool. Firstly, the degree to which assumptions were met and whether the method used in the study was appropriate. Secondly, the validation of the statistical model presented including whether the model had been validated using fresh data allowing an evaluation of generalizability. Thirdly, how adequately the authors reported the analysis procedures used and the results.

The quality assessment tool was based on the Statistical Analyses and Methods in the Published Literature (SAMPL) guidelines [30]. Items from the guidelines were rewritten as questions and additional items were added when required (Table 1). The tool was divided into three sub-sections. The "Reporting of statistical methods" sub- 
Table 1 Quality assessment tool

Sub-section Items
and area of
interest

Section 1-Reporting of statistical analysis

Data quality Does the study report information about the research population?

Does the study report what type of data has been used for the analysis, whether it is primary data (collected initially for the study) or secondary data (from a different source)?

If the study used secondary data does the study name, the databases which have been used for the analysis?

If the study has used different databases, does the study describe any linkage between the databases?

Does the study report whether the data set is a representative data set?

Preliminary Does the study report any statistical procedures used to modify raw data before the analysis?

analyses

Primary Does the study describe about the purpose of the analysis?

analyses Does the study identify the variables used in the analysis and summarize each with descriptive statistics?

Does the study describe the main methods fully, for analyzing the primary objectives of the study?

Is the study clear on which method is used for each analysis, rather than just listing all statistical methods used in one place? If the method includes any assumptions, does the study verify that the data conformed to the assumptions of the test used to analyze them?

Does the study indicate whether and how any allowance or adjustments were made for multiple comparisons (performing multiple hypothesis tests on the same data)?

Does the study report how it deals with missing data?

If relevant, does the study report how any outlying data were treated in the analysis?

Does the study report the alpha level (e.g., 0.05) that defines statistical significance?

Does the study report the name of the statistical package or program used in the analysis?

If the study needs to control any variables for its objective, does the study report it properly?

Supplementary Does the study describe sensitivity analyses if applicable?

analyses Does the study test for the underlying assumptions of the methods used in the analysis?

Does the study identify post hoc analyses, including unplanned subgroup analyses, as exploratory?

If there is an imbalance that exists in the outcome variable of the data set, does the study report how the training phase

overcome this issue?

Section 2-Reporting of results

Reporting Does the study report numbers-especially measurements-with an appropriate degree of precision. For ease of

numbers and comprehension and simplicity, rounded to a reasonable extent?

descriptive stat Does the study report total sample and group sizes for each analysis?

avg Does the study report numerators and denominators for all percentages?

Does the study summarize data that are approximately normally distributed with means and standard deviations (SD)?

Use the form: mean $(S D)$, not mean \pm SD?

Does the study summarize data that are not normally distributed with medians and interpercentile ranges, ranges, or both (report the upper and lower boundaries of interpercentile ranges and the minimum and maximum values of ranges, not just the size of $t)$ ?

Does the study report the variability of the data set using either standard deviations, inter-percentile ranges, or ranges (the SE is an inferential statistic - it is about a 68\% confidence interval — not a descriptive statistic)?

Does the study display summarized or exact data in tables?

Does the study display data in figures? Tables present exact values, and figures provide an overall assessment of the data?

Reporting risk Does the study describe the type of rate (e.g., incidence rates; survival rates), ratio (e.g., odds ratios; hazard ratios), or risk (e.g., and ratios absolute risks; relative risk differences), being reported?

Does the study describe the quantities represented in the numerator and denominator?

Does the study report the time period over with each rate applies?

Does the study report any unit of population (that is, the unit multiplier: e.g., $\times 100 ; \times 10,000$ ) associated with the rate?

Does the study consider reporting a measure of precision (a confidence interval) for estimated risks, rates, and ratios?

Validation Does the study describe methods of validation used in the training phase (e.g., cross validation, use of test/hold-out sample)? Does the study describe the attempts to generalize the model beyond the immediate context?

Section 3-Method specific quality indicators

Regression Does the study describe the purpose of the analysis?

analysis Does the study confirm that the assumptions of the analysis were met? For example, in linear regression indicate whether an analysis of residuals confirmed the assumptions of linearity.

Does the study report the regression equation for either simple or multiple (multivariable) regression analyses?

For primary comparisons analyzed with simple linear regression analysis, does the study consider reporting the results graphically,

in a scatter plot showing the regression line and its confidence bounds?

Does the study report the alpha level used in the univariate analysis?

Does the study report whether the variables were assessed for collinearity?

Does the study report whether variables were assessed for interactions?

Does the study describe the variable selection process by which the final model was developed (e.g., forward stepwise; best subset). 
Table 1 Quality assessment tool (Continued)

\begin{tabular}{|c|c|}
\hline $\begin{array}{l}\text { Sub-section } \\
\text { and area of } \\
\text { interest }\end{array}$ & Items \\
\hline & $\begin{array}{l}\text { Does the study report the regression coefficients (beta weights) of each explanatory variable and the associated confidence } \\
\text { intervals and } P \text { values, preferably in a table? } \\
\text { Does the study provide a measure of the model's "goodness-of-fit" to the data (the coefficient of determination, } r 2 \text {, for simple } \\
\text { regression and the coefficient of multiple determination, R2, for multiple regression)? }\end{array}$ \\
\hline Survival analysis & $\begin{array}{l}\text { Does the study describe the purpose of the analysis? } \\
\text { Does the study describe the dates or events that mark the beginning and the end of the time period analyzed? } \\
\text { Does the study specify the circumstances under which data were censored? } \\
\text { Does the study specify the statistical methods used to estimate the survival rate? } \\
\text { Does the study confirm that the assumptions of survival analysis were met? } \\
\text { For each group, give the estimated survival probability at appropriate follow-up times, with confidence intervals, and the number } \\
\text { of participants at risk for death at each time. It is often more helpful to plot the cumulative probability of not surviving. } \\
\text { For each group, give the estimated survival probability at appropriate follow-up times, with confidence intervals, and the number } \\
\text { of participants at risk for death at each time. It is often more helpful to plot the cumulative probability of not surviving. } \\
\text { Reporting median survival times, with confidence intervals, is often useful to allow the results to be compared with those of other } \\
\text { studies? } \\
\text { Does the study present the full results in a graph (e.g., a Kaplan-Meier plot) or table? } \\
\text { Does the study specify the statistical methods used to compare two or more survival curves? } \\
\text { Does the study report the } P \text { value, when comparing two or more survival curves with hypothesis tests? } \\
\text { Does the study report the regression model used to assess the associations between the explanatory variables and survival or time- } \\
\text { to-event? } \\
\text { Does the study report a measure of risk (e.g., a hazard ratio) for each explanatory variable, with a confidence interval? }\end{array}$ \\
\hline SEM models & $\begin{array}{l}\text { Does the study report all the parameters and their standard errors? } \\
\text { Does the study report the reason for the choice of a clear and complete form of path model structure? } \\
\text { Does the study report the global indices of fit? } \\
\text { Does the study provide reasons as justification for omitted directed and non-directed arcs? } \\
\text { Does the study report alternative and equivalent models? }\end{array}$ \\
\hline
\end{tabular}

section included assessments of data quality (5 items), preliminary analyses (1 item), primary analyses (11 items), and supplemental analyses (4 items). The "Reporting of results" sub-section was made up of items assessing the reporting of numbers, descriptive statistics and averages (8 items), reporting risk ratios (5 items), and validation (2 items). The third section assessed "Method specific quality indicators" for regression analysis (10 items), survival analysis (13 items), and structural equation modeling (5 items). Studies were not assessed on items irrelevant to the methods used.

\section{Quality assessment procedure}

Included studies were quality assessed by two independent authors (SM and SS) using the purpose-made quality assessment tool. The quality assessment tool was completed as a Google doc and automatically exported into an Excel file for analysis. Disagreements were resolved by a third author (DM). Items were allocated a score of 1 for yes, 0 for no, or NA for not applicable with higher quality papers receiving higher quality scores. Subsection quality scores were calculated by averaging responses to reveal a quality score ranging from 1 when all quality indicators were met, to 0 when no quality indicators were met. When an item was not applicable, that item was excluded from the mean score of that study's rating. Then each reviewers' sub-section quality scores were averaged to get a final quality score for every study independently. Finally, the independently reviewed quality scores of the two reviewers were averaged to get an overall quality measure for each study. Studies were then categorized as scoring low (0 to 0.333$)$, medium (0.334 to 0.666$)$, and high $(0.667$ to 1.00$)$; i.e., $1 / 3=0.333$ with one being the totality of all studies included in the review.

\section{Results}

The flow of studies from identification, through screening for eligible inclusion, to the final synthesis, is presented in the PRISMA flowchart, Fig. 1. One thousand one hundred and five records were identified. Of the 252 studies included in the full-text screen (Appendix S4), 243 were identified in the database search and nine were identified in the forwards and backward search. Agreement between the two reviewers (RS and SM) of the full texts for inclusion was low $(k=0.469, p<0.00)$. These disagreements were resolved by a third author (SM), resulting in twenty studies being included in the quality assessment, comprising of data from a total of 2,379,862 individuals. The statistical techniques, findings, and characteristics of the included studies are presented in Table 2.

\section{Vehicle type and population}

Nine out of the 20 reviewed studies included all vehicle types (i.e., cars, motorcyclists, and trucks). The 


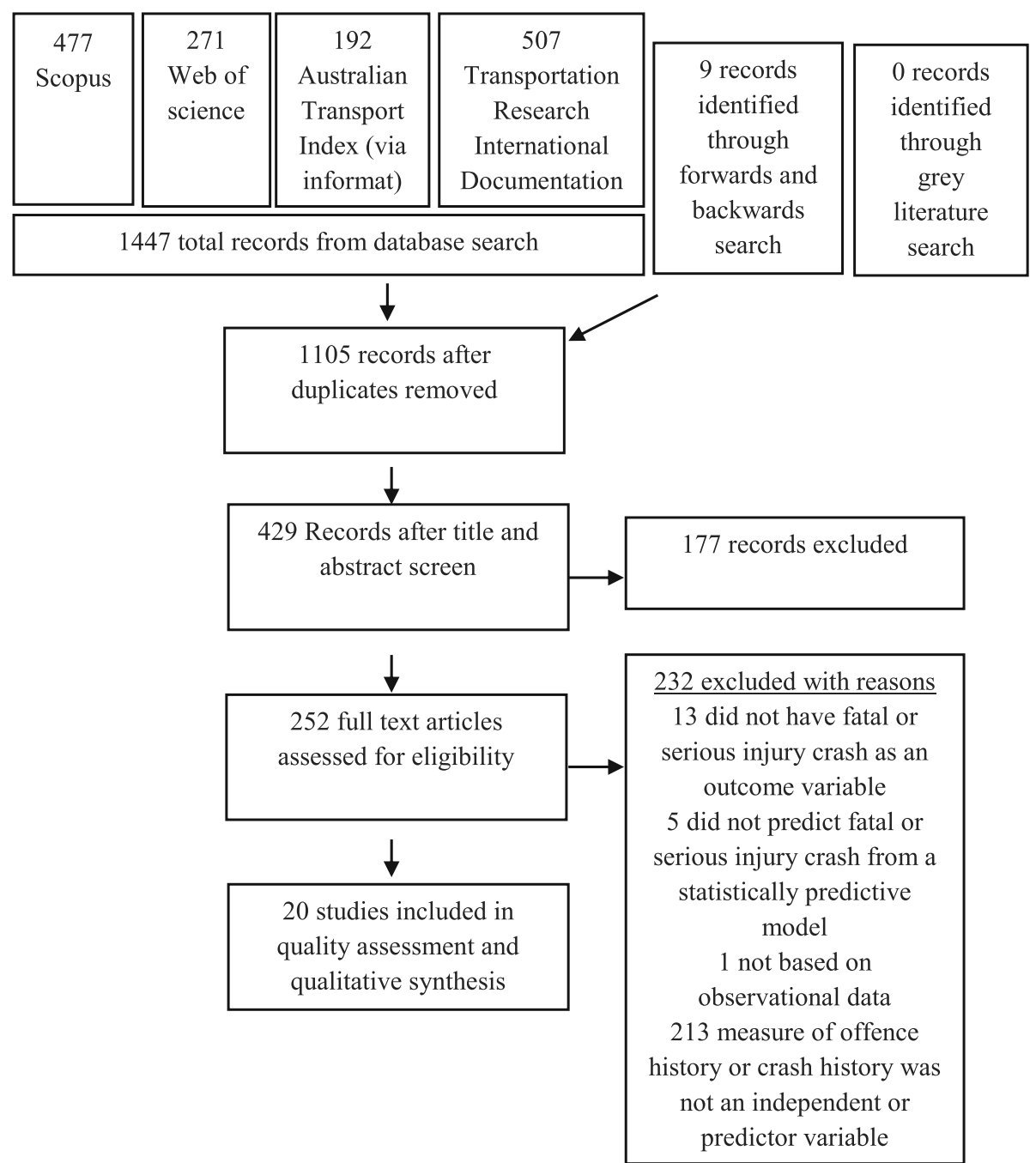

Fig. 1 PRISMA flowchart

remaining 11 studies explored the prediction of FSI crashes from offense history and crash history using data related to distinct vehicle types: buses (1 study), heavy trucks (5 studies), and cars and light trucks (4 studies). Seventeen studies used data representing the population, three countries were represented-USA, Finland, and Canada, and two states were represented-North Dakota (USA) and South Australia (Australia). Of the studies using sample data, one used a $20 \%$ representative sample based on Israeli census data, one used data from six states in the USA, and a third study selected various highways within the USA state of Wyoming (Appendix S5).

\section{Quality assessment}

Overall, there was high agreement between the two reviewers of statistical quality (inter-class correlation coefficient 0.919; Appendix S6). Fifteen studies used logistic regression and most of these studies received quality scores in the middle range (low $=3$, middle $=12$, high $=$ 2 ). The only study to use structural equation modeling also received a quality score in the middle range. One study used a survival analysis, and one used a Poisson multi-level growth curve model, both receiving a highquality score. Quality scores tended to be consistently higher for more recently published studies (Table 3 ). The improvement in quality seems to be led predominantly by improved "Reporting of statistical methods," while the method specific quality indicators provide the poorest results (Fig. 2).

\section{Qualitative synthesis of study outcomes}

Using binary logistic regression analyses, seven studies reported that offense history predicts FSI crash involvement. Perneger and Smith [32] found that having an invalid driving license or license suspension, or a prior DWI (driving while intoxicated) conviction increases the 


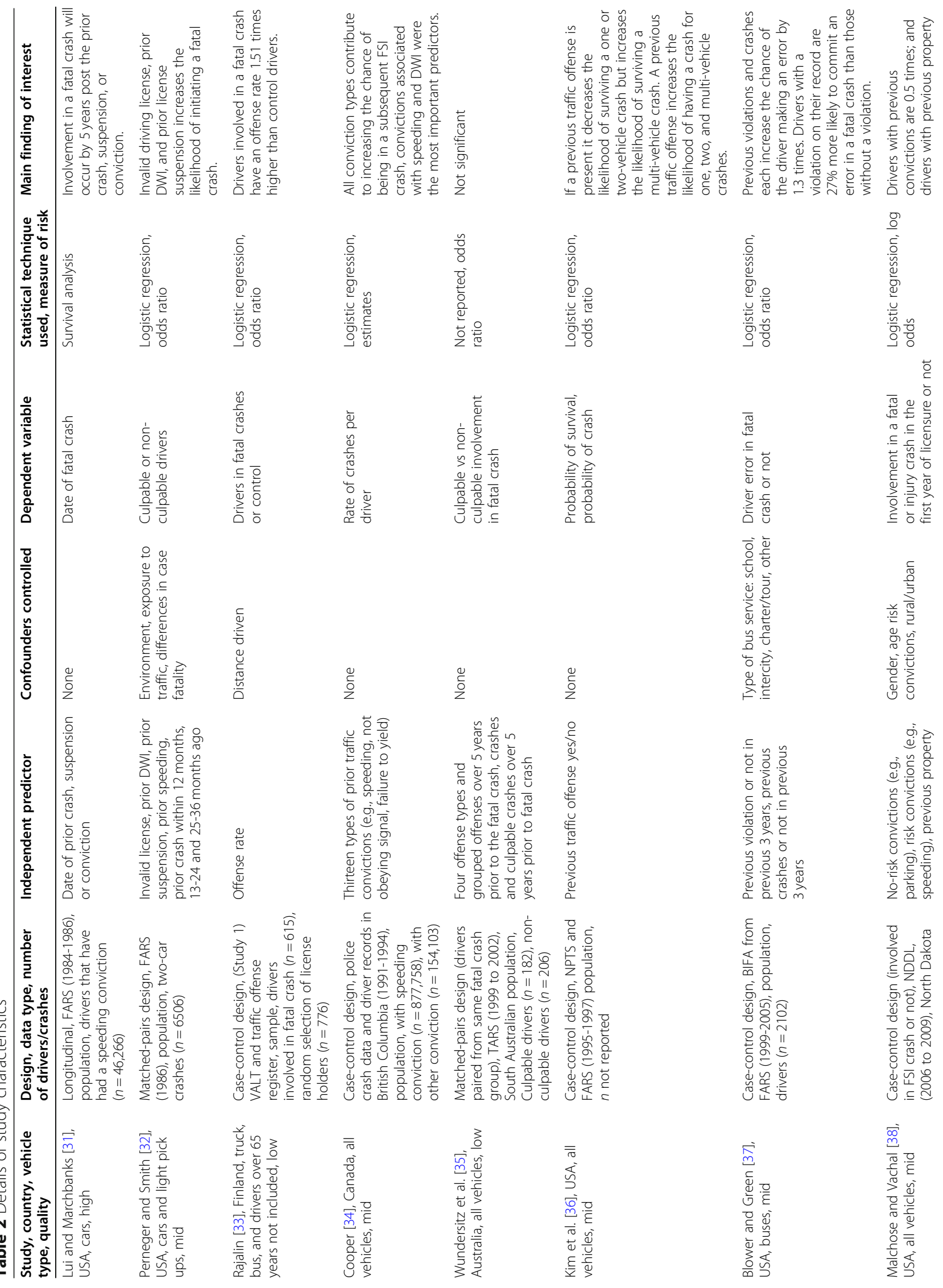




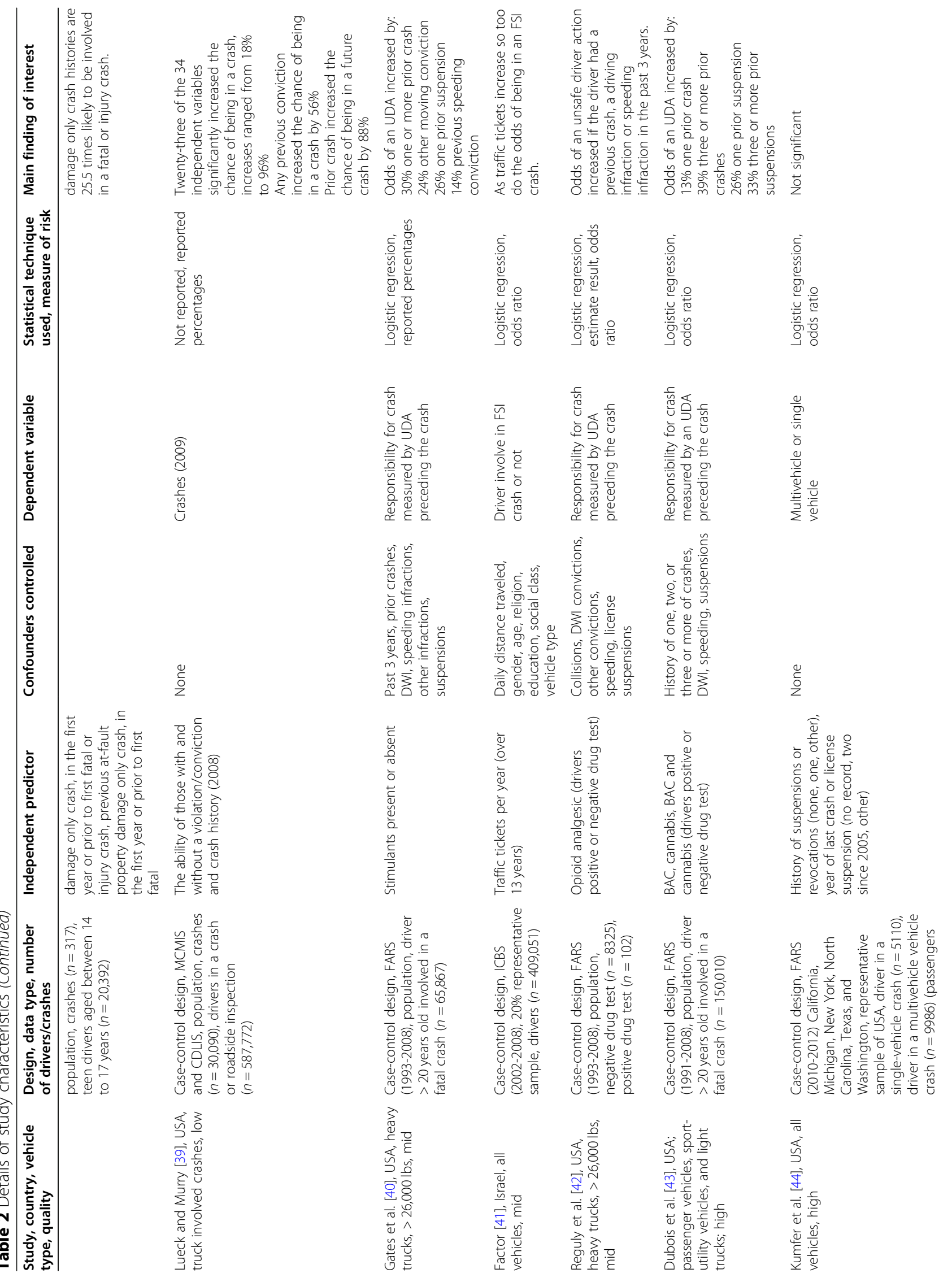




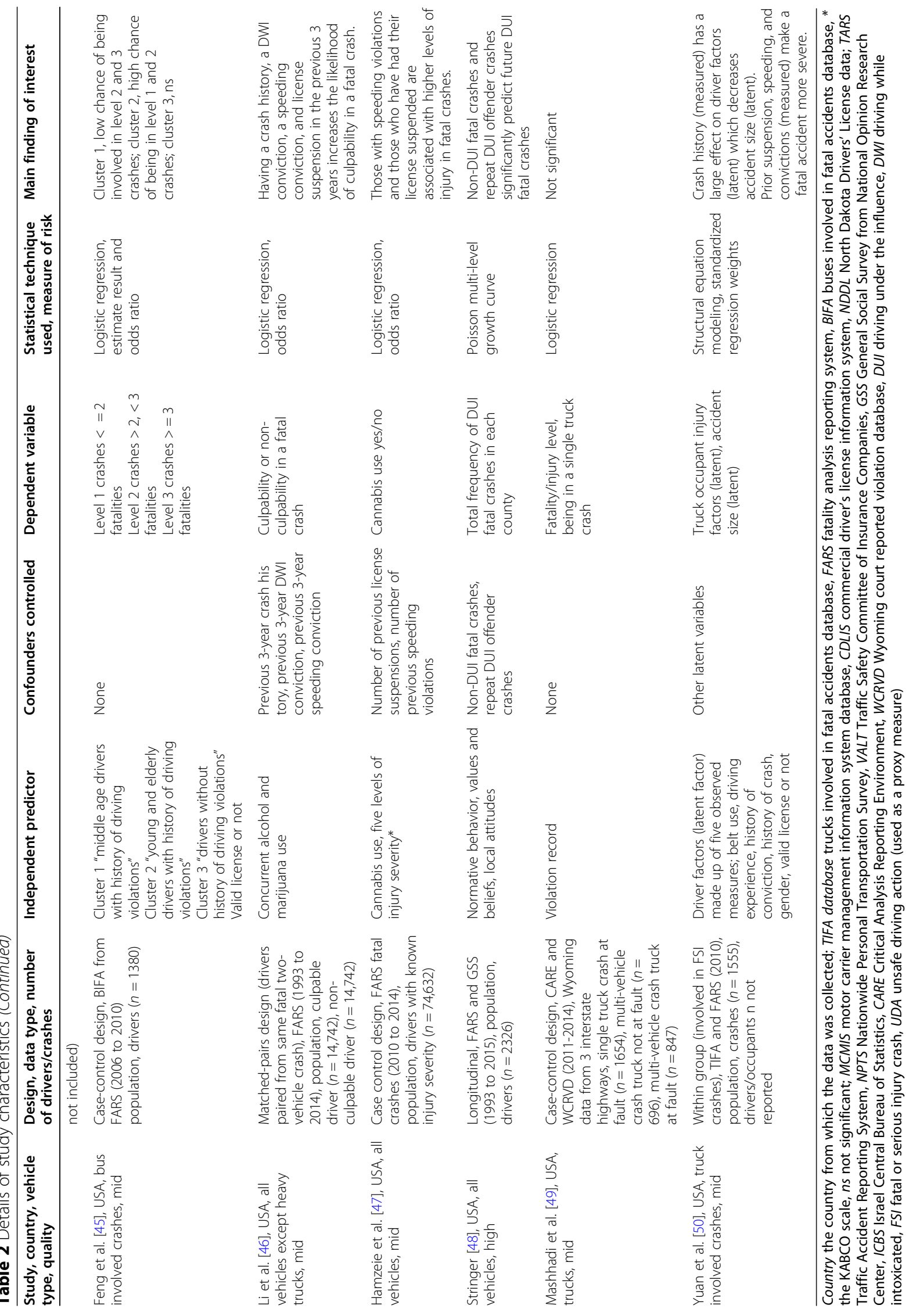


Table 3 Quality tool assessment results

\begin{tabular}{|c|c|c|c|c|}
\hline Studies grouped by statistical technique & $\begin{array}{l}\text { Reporting of statistical } \\
\text { methods }\end{array}$ & $\begin{array}{l}\text { Reporting of statistical } \\
\text { results }\end{array}$ & $\begin{array}{l}\text { Method specific quality } \\
\text { indicators }\end{array}$ & $\begin{array}{l}\text { Overall quality } \\
\text { score }\end{array}$ \\
\hline \multicolumn{5}{|l|}{ Logistic regression } \\
\hline Perneger and Smith 1991 & 0.704 & 0.552 & 0.299 & 0.463 \\
\hline Rajalin 1994 & 0.193 & 0.391 & 0.000 & 0.146 \\
\hline Cooper 1997 & 0.372 & 0.188 & 0.444 & 0.362 \\
\hline Wundersitz et al. 2004 & 0.435 & 0.525 & 0.167 & 0.323 \\
\hline Kim et al. 2006 & 0.470 & 0.432 & 0.278 & 0.365 \\
\hline Blower and Green 2010 & 0.675 & 0.590 & 0.556 & 0.594 \\
\hline Malchose and Vachal 2011 & 0.646 & 0.449 & 0.500 & 0.524 \\
\hline Lueck and Murry 2011 & 0.333 & 0.382 & 0.111 & 0.234 \\
\hline Gates et al. 2013 & 0.741 & 0.617 & 0.611 & 0.645 \\
\hline Factor 2014 & 0.880 & 0.576 & 0.389 & 0.558 \\
\hline Reguly et al. 2014 & 0.907 & 0.636 & 0.556 & 0.664 \\
\hline Dubois et al. 2015 & 0.869 & 0.750 & 0.611 & 0.710 \\
\hline Kumfer et al. 2015 & 0.938 & 0.651 & 0.611 & 0.703 \\
\hline Feng et al. 2016 & 0.750 & 0.617 & 0.500 & 0.592 \\
\hline Li et al. 2017 & 0.697 & 0.464 & 0.997 & 0.755 \\
\hline Hamzeie et al. 2017 & 0.741 & 0.571 & 0.500 & 0.578 \\
\hline Mashhadi et al. 2018 & 0.696 & 0.569 & 0.500 & 0.566 \\
\hline \multicolumn{5}{|l|}{ Structural equation modeling } \\
\hline Yuan et al. 2019 & 1.000 & 0.387 & 0.600 & 0.647 \\
\hline \multicolumn{5}{|l|}{ Survival analysis } \\
\hline Lui and Marchbanks 1990 & 0.481 & 0.625 & 0.808 & 0.680 \\
\hline \multicolumn{5}{|l|}{ Poisson multi-level growth curve } \\
\hline Stringer 2018 & 0.958 & 0.504 & 0.778 & 0.755 \\
\hline
\end{tabular}

likelihood of initiating a fatal crash. Cooper [34] found that fourteen conviction types increase the chance of being in a subsequent FSI crash; speeding and DWI convictions were the most important. These findings are supported by Factor [41], who used traffic infringement tickets as a predictor variable, and Rajalin [33], who compared drivers who had had an FSI crash to those who had not. Further nuance to the role of offense history is added by Kim et al. [36]. Offense history increased the likelihood of having a crash regardless of the number of vehicles involved. Additionally, those with previous traffic offenses were less likely to survive a one or two-vehicle crash, but more likely to survive a crash involving three or more vehicles. Speeding violations and a license suspension, rather than any other type of offense, were associated with increased injury severity [47].

Six studies using binary logistic regression reported that both crash history and offense history increase the likelihood of FSI crash involvement. The odds of a driver making an error directly leading to an FSI crash increased by approximately $27 \%$ if they had been responsible for a previous crash, a driving infraction/violation, or speeding infraction [37, 38, 42]. Specifically, driver error leading to an FSI crash increased by $13 \%$ if the driver had one prior crash [43], 30\% if the driver had one or more prior crashes [40], and 39\% if the driver had three or more prior crashes [43]. Prior license suspension was a strong predictor of driver error leading to an FSI crash: one suspension increased the odds by $26 \%$ $[40,43]$ and three or more prior suspensions increased the odds by $33 \%$ [43]. Not only are offense history and crash history associated with driver errors causing an FSI crash, but they are also associated with the likelihood of the driver being legally culpable. Predictors of being legally culpable include a crash history, a DWI conviction, a speeding conviction, and a license suspension in the 3 years prior to the crash [46]. Property damage only crashes also predicted FSI crash involvement [38]. Feng et al. [45] took a unique approach by grouping drivers on several characteristics. Middle-aged drivers with a history of convictions had a low chance of being involved in a crash involving more than two fatalities. Young and elderly drivers with a history of 


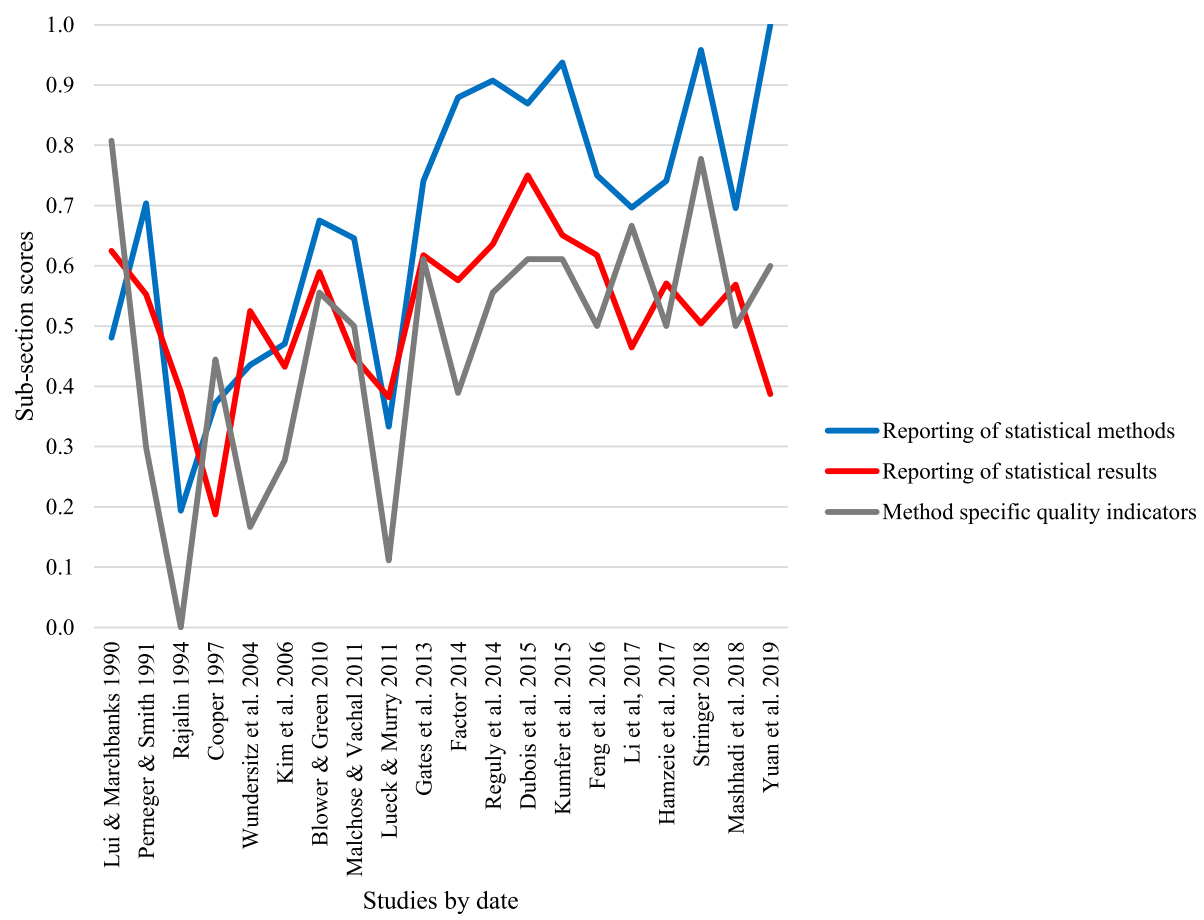

Fig 2 Quality assessment sub-section scores for included studies

violations had a high chance of being involved in a crash involving less than 3 fatalities [45].

Analyses other than logistic regression produced comparable findings. Based on longitudinal data, Stringer [48] found that crash history and offense history significantly predict future fatal crashes with crash history being the more important predictor. Yuan et al. [2019] found that prior suspension and speeding convictions are associated with fatal accidents. Finally, Lui and Marchbanks [31] found that should a fatal crash occur after license suspension, a conviction, or prior crash, it is likely to occur within 5 years. While the predictors of FSI crashes have been identified, these findings cannot be verified given that most of these studies were assessed as having only low to middle quality in our quality assessment.

\section{Critical discussion}

\section{Strengths of logistic regression in this context}

There are several reasons for the popularity of logistic regression. Logistic regression has many different forms allowing its use for conditional logistic regression with matched-pairs data [32, 45]; ordinal and nominal logistic regression when the dependent variable has several categories [45, 47]; direct logistical regression when no predictor variables are considered more or less important than the others [37]; sequential logistic regression when confounding variables need to be controlled [41, 43]; stepwise logistic regression when an exploratory approach is needed [49]; and censored regression when data for the dependent variable is incomplete [36]. Further, the assumptions for logistic regression are lenient. Also, logistic regression can perform several functions. It can predict group membership, identify important predictors, identify interactions among predictors and provide odds ratios for quantifying the effects of predictors. Moreover, the accuracy of logistic regression models is easily assessed using a variety of measures including the proportion of variance in the dependent variable explained by the predictors, using pseudo $R$ squared values.

\section{Weaknesses of the use of logistic regression in this review}

Most of the papers that applied a logistic regression model in this review was rated as "medium" quality. There were striking statistical deficiencies found in these studies. Firstly, the selection of predictor variables and the number of predictors included often appeared to be made post hoc, i.e., inclusion was justified only after the model was created. Given the scope of variables available from large population databases recording fatal crashes, a surprising observation was that only five studies [32, 37, 40, 43, 49] described the variable selection process used. While the practice of post hoc justification of predictors is common, it is damaging to the integrity of findings and real-world implications. Logistic regression is often used to inform life and death decisions; therefore, inadequate or poor a 
priori variable selection may indeed lead to unwanted consequences.

Secondly, of vital importance to the quality of analyses and subsequent confidence in findings is the adherence to underlying statistical assumptions. Only two studies using logistic regression confirmed that the assumptions were met $[46,47]$. Particularly problematic within the studies using logistic regression was the failure to check for correlations between predictor variables (multicollinearity) and interactions. Of studies using logistic regression, only two studies reported checking multicollinearity $[44,46]$, and only five studies, checked for interactions $[40,42-44,46]$. The presence of multicollinearity and interactions within the predictor variables artificially increases their ability to predict FSI crashes and makes interpretation of odds ratios problematic. Not meeting these assumptions raises questions about the validity of the results.

A critical observation repeatedly made by reviewers of statistical models is the inclusion of unwanted correlations in data. Examples are, serial correlation, i.e., correlation over time [18], spatial and temporal correlation between predictor variables, and correlation between predictor variables and error [20]. The inclusion of these correlations in data results in incorrect inferences being made from the results. To illustrate the problem, helmet use may appear to reduce crash fatalities, yet when a biological or psychological predisposition to risk predicts both helmet use and crash involvement, helmet use may no longer be a significant predictor of fatalities. This argument is supported by a clear relationship between sensation seeking and risky driving [51]. The problem of unwanted correlation in the data is relevant in this review, when contemporaneous offense and crash data are considered. It is important to note that there is likely to be a two-way relationship between offense history and crash history in this context. Not only is someone with an extensive offense history more likely to have a crash. In addition, it is likely that a crash caused by a driver will lead to the driver being charged with an offense [49].

The third weakness identified in the reviewed studies using logistic regression is the lack of validation. For example, none of the studies reported the results of a Hosmer Lemeshow goodness of fit test or areas under the receiver operating characteristic curve. Validation of final models using new data and the use of validation during model creation was not conducted anywhere in the reviewed literature. Given the pervasive international interest and enormous volume of publications on FSI crashes, the lack of validated models that predict FSI crashes from offense history, crash history, and licensing variables is an important limitation. However, one of the papers in this review [46] did conduct two sensitivity analyses for their models. One of these sensitivity analyses tested their model with data from the USA states that accounted for more than $80 \%$ of FSI crashes in the USA and the second sensitivity analysis tested the model on data for two different time periods.

The fourth major limitation is lack of effective calibration of the models, considering any imbalance in the data in order to provide accurate estimates of classification accuracy. Indeed, no study in this review presented a confusion/classification matrix to evaluate how well the model classified participants into the correct categories. Both validation and calibration must be conducted after a model has been created to confirm its classification accuracy [52].

Taken together these four deficits within the literature using logistic regression models, drastically lowers confidence in the overall findings that offense history or crash history can accurately predict future FSI crashes.

\section{Critical summary of other modeling techniques utilized in this review}

Three studies used a modeling technique other than logistic regression: survival analysis [31], Poisson multilevel growth curve modeling [48], and structural equation modeling [50]. The use of survival analysis was largely responsible for Lui and Marchbanks article [31] receiving a high-quality score, with many statistical considerations not being applicable. However, lacking within the study was a description of how raw data was treated, whether preliminary analyses were conducted, and the reporting of descriptive statistics. These limitations reduce the generalization and utility of the model.

A Poisson multi-level growth curve model was used by Stringer [48] to address the question, "What is the probability that in a given period an FSI crash will occur?" Stringer [48] received the highest quality score among the included studies, suggesting that this paper was the most rigorous. The weaknesses identified in Stringer [48] did not seriously undermine the validity of the results. The most important result was based on longitudinal data; crash history and offense history significantly predict future fatal crashes with crash history being the more important predictor. Weaknesses were failure to report the statistical packages used and descriptive statistics for the included variables. In addition, there was no model validation attempt so the extent to which the model has general application is unknown. Notable strengths of the study were the use of a Poisson distribution to avoid overestimation of zero values, the use of longitudinal data, and rigorous checks of the model assumptions.

Yuan et al. [50] used structural equation modeling to make sense of a broad range of variables involved in FSI crashes. Structural equation modeling allows for the modeling of latent constructs using measurement 
models and for path models used to assess relationships between variables that are measured (observed) or latent (not observed) or both. An example of a latent variable constructed in this paper is "Driver Factors" made up from the following measured variables-seatbelt use, driving expertise, offense history, crash history, and gender (measurement model). "Driver Factors" was used to predict two other latent variables, "Truck Occupant" and "Accident Size," using a path model. This technique was appropriately used by Yuan et al. (2019) as they had a large data set, appropriate measures for the latent variables which met the underlying model assumptions, and appropriate values for the goodness of fit indices.

\section{Recommendations for future research of FSI crashes using offense history and crash history as predictor variables}

There are multiple recommendations for future statistical modeling of FSI crashes from offense and crash history based on the reviewed literature. Firstly, when using population data, and data containing a large number of variables, the use of statistical significance indicators such as p-values are inappropriate. For example, tests of predictor coefficients will inevitably result in multiple type 1 errors. Instead, researchers need to follow and report rigorous methods of variable selection prior to commencing the main analysis. Secondly, future research needs to test and report the checking of statistical assumptions and models need to be validated using fresh data. In addition, classification accuracy needs to be reported considering any imbalance in the crash data. The underreporting of crashes has previously been argued to create a non-random data set, violating traditional statistical assumptions [20], but this is unlikely for FSI crashes.

Thirdly, machine learning should be utilized to overcome the problem of unwanted correlations in the data when there are very large sample sizes. These methods do not rely on $p$ values for model selection purposes. For example, random forest can accommodate interactions between predictor variables and can sort the predictor variables according to their importance [21]. Importance ranking is crucial when there are many predictor variables and there is a need for organizations to identify the most important variables for policy changes. Similarly, to random forest models, gradient boosting algorithms may be useful in that they can handle large sets of predictor variables and interactions. The advantage of these models is their ability to more accurately classify minority classes. However, careful considerations for the tuning of the parameters during the training phase, often involving cross-validation, is needed in order to avoid overfitting. Unfortunately, interpretation of random forests and gradient boosting is difficult. This makes the results harder to explain to non-technical audiences compared to the results of traditional statistical models such as logistic regression models which provide odds ratios with $95 \%$ confidence intervals.

Another prominent issue faced by researchers analyzing FSI crash data is the prevalence of highly imbalanced data sets with respect to the class of the dependent variable. Most drivers have no history of being involved in an FSI crash. This imbalance in the number of drivers who have and who have not been involved in an FSI crash is problematic for statistical models attempting to classify drivers as being at risk of FSI crash involvement as models developed using imbalanced data tend to struggle to correctly classify those in the minority class (i.e., drivers most at risk of FSI crash involvement). In order to overcome this problem, researchers should consider using techniques such as the synthetic minority over sampling technique (SMOTE) which allows oversampling of the minority class in a representative manner. Another consideration for researchers is the use of hidden Markov models or recurrent neural networks to identify drivers more a risk of an FSI crash. These methods could use the past sequence of offenses to predict the probability of a future FSI crash.

Finally, there is a need for the use of statistical quality assessment tools in future reviews of the crash literature. It is hoped that future reviews of the FSI and broader crash and transportation literature will refine and use our tool to assess the quality of statistical analyses. This is important because a failure to identify poor statistical analysis in reviews may lead to incorrect conclusions and misinformed policy. The use of a standardized assessment tool improves the objectivity of a review's findings. Such tools are easy and quick to use, also allowing comparisons between reviewer scores and ensuring that all studies are assessed on the same characteristics.

\section{Limitations of this review}

Language and geographical bias limited this review. Similar to other reviews of related topics [20, 53], the bulk of the research was conducted in the USA. We considered only articles written in English and the grey literature review was confined to higher-income countries with lower road death rates. The review was limited to a qualitative synthesis of included studies as a metaanalysis was deemed inappropriate. This was because most models reviewed included many predictor variables in addition to our predictors of interest, namely, offense history and crash history. Further limitations were the time and scope of the search protocol. Despite searching four prominent databases, it is possible that further applicable publications were missed. The lack of search terms for specific offenses (e.g., "speeding," "drink driving") may have limited the identification of additional 
studies present in the databases that were searched. Additionally, one author conducted the screening of identified records and abstracts potentially introducing bias and missed studies. The applicability of our findings is limited to high income developed countries, as FSI crashes are not rare and offense history may not be reliable in countries with low and middle incomes.

\section{Conclusions}

This review contributed to the literature in multiple ways. The study developed a statistical quality assessment tool and demonstrated how it can be utilized when presenting evidence of FSI crash prediction. The review identified that multicollinearity, model validation, and appropriate methods for the selection of predictor variables remain problematic in studies predicting FSI crashes from offense and crash history. However, the most recent studies reported more rigorous modeling practices. Future studies modeling FSI crash risk using offense and/or crash history should consider employing machine learning methods to overcome some of the key limitations of the traditionally used statistical techniques identified in this review. Seven out of the 15 studies using logistic regression reported an association between offense history and FSI crashes. Suspension bans and crash history were also commonly reported as having an association with FSI crashes.

\section{Supplementary information}

Supplementary information accompanies this paper at https://doi.org/10. 1186/s13643-020-01475-7.

Additional file 1. Data base search strings 7/6/2019. Appendix S1-S6

\begin{abstract}
Abbreviations
FSI: Fatal and serious injury; PRISMA: Preferred Reporting Items for Systematic Reviews and Meta-analyses; PROSPERO: International prospective register of systematic reviews; ATRI: Australian transport index; ITRD: Transportation Research International Documentation database; SAMPL: Statistical Analysis and Methods in the Published Literature; SM: Samuel Muir; SS: S. S. M. Silva; DM: Denny Myer; TIFA database: Trucks Involved in Fatal Accidents database; FARS: Fatality Analysis Reporting System; BIFA: Buses Involved in Fatal Accidents database; KABCO scale: Fatal, incapacitating injury, nonincapacitating injury, possible injury, property damage only scale; MCMI S: Motor Carrier Management Information System database; CDLI S: Commercial Driver's License Information System; NDDL: North Dakota Drivers' License data; TARS: Traffic Accident Reporting System; NPTS: Nationwide Personal Transportation Survey; VALT: Traffic Safety Committee of Insurance Companies; GSS: General Social Survey from National Opinion Research Center; ICBS: Israel Central Bureau of Statistics; CARE: Critical Analysis Reporting Environment; WCRVD: Wyoming Court Reported Violation Database; DUI: Driving under the influence; DWI: Driving while intoxicated; UDA: Unsafe driving action; USA: United States of America
\end{abstract}

\section{Acknowledgements}

There are no additional contributors to acknowledge.

\section{Authors' contributions}

All authors contributed to writing the manuscript, screening studies, conducting analysis, and design. All authors read and approved the final manuscript.

\section{Funding}

This work was supported by VicRoads, part of the Department of Transport, Victoria. VicRoads outlined the central research question, but all other aspects of the review were conducted independently.

Availability of data and materials

All data analyzed during this study are included in this published article and its supplementary contents.

Ethics approval and consent to participate

All data originated from published articles.

\section{Consent for publication}

No individual person's data was presented.

\section{Competing interests}

No competing interests are reported by the authors.

Received: 2 April 2020 Accepted: 3 September 2020

Published online: 28 September 2020

\section{References}

1. AustLII. Transport Accident Act 1986 - Schedule [Internet]. Victorian current acts. 2019 [cited 2019 Oct 7]. Available from: http://www.austlii.edu.au/au/ legis/vic/consol_act/taa1986204/sch1.html.

2. Bureau of Infrastructure Transport and Regional Economics. Road trauma Australia 2016 statistical summary. In: Canberra ACT; 2017.

3. Australian Bureau of Statistics. Criminal Courts, Australia, 2017-18 [Internet]. 4513.0 Criminal Courts, Australia, 2017-18. 2019 [cited 2019 Oct 7]. Available from: https://www.abs.gov.au/ausstats/abs@.nsf/mf/4513.0.

4. Kraus J, Peek C, McAethur D. The effect of the 1992 California motocycle helment use law on motorcycle crash fatalities and injuries. JAMA. 1994; 272(19):1506-11.

5. Wali B, Ahmed A, labal S, Hussain A. Effectiveness of enforcement levels of speed limit and drink driving laws and associated factors - exploratory empirical analysis using a bivariate ordered probit model. J Traffic Transp Eng (English Ed [Internet]. 2017;4(3):272-9. Available from: https://doi.org/10. 1016/j.jtte.2017.04.001.

6. Yang B, Liu P, Chan C, Xu C, Guo Y. Identifying the crash characteristics on freeway segments based on different ramp influence areas. Traffic Inj Prev [Internet]. 2019;20(4):386-391. Available from: https://doi.org/10.1080/ 15389588.2019.1588965.

7. Chen Y, Liu G, Zhang Z, Hou S. Integrated design technique for materials and structures of vehicle body under crash safety considerations. Struct Multidiscip Optim. 2017;56(2):455-72.

8. Salmon PM, Read GJM, Beanland V, Thompson J, Filtness AJ, Hulme A, et al. Bad behaviour or societal failure? Perceptions of the factors contributing to drivers' engagement in the fatal five driving behaviours. Appl Ergon [Internet]. 2019;74(April 2018):162-71. Available from: https://doi.org/10. 1016/j.apergo.2018.08.008.

9. Asbridge M, Hayden JA. Acute cannabis consumption and motor vehicle collision risk: systematic review of observational studies and meta-analysis. BMJ. 2012;536(February):1-9.

10. Asgarian F, Namdari M, Soori $\mathrm{H}$. Worldwide prevalence of alcohol in fatally injured motorcyclists: a meta-analysis. Traffic Inj Prev. 2019.

11. Bingham CR, Ehsani JP. The relative odds of involvement in seven crash configurations by driver age and sex. J Adolesc Health [Internet. 2012;51(5): 484-90 Available from: https://doi.org/10.1016/j.jadohealth.2012.02.012.

12. Moradi A, Saeed S, Nazari H, Rahmani K. Sleepiness and the risk of road traffic accidents: a systematic review and meta-analysis of previous studies. Transp Res Part F Psychol Behav [Internet]. 2018; Available from: https://doi. org/10.1016/j.trf.2018.09.013.

13. DeYoung DJ, Gebers MA. An examination of the characteristics and traffic risks of drivers suspended/revoked for different reasons. J Saf Res. 2004;35: 287-95.

14. Elliott MR, Waller PF, Raghunathan TE, Shope JT. Predicting offenses and crashes from young drivers' offense and crash histories. Traffic Inj Prev. 2007; 6586.

15. Rezapour M, Wulff S, Ksaibati K. Predicting truck at fault crashes using crash and traffic offence data. Open Transp J. 2018;12:128-38. 
16. Sagberg F, Economics T, Engström J, Group V. A review of research on driving styles and road safety. Hum Factors. 2015;57(7):1248-75.

17. Leal NL, Watson BC. The road safety implications of illegal street racing and associated risky driving behaviours: an analysis of offences and offenders. Accid Anal Prev [Internet]. 2011;43(4):1547-1554. Available from: https://doi. org/10.1016/j.aap.2011.03.010.

18. Noland RB, Karlaftis MG. Sensitivity of crash models to alternative specifications. Transp Res Rec Part E Logist Transp Rev. 2005;41(5):439-58.

19. Venkataraman N, Shankar V, Blum J, Hariharan B, Hong J. Transferability analysis of heterogeneous overdispersion parameter negative binomial crash models. Transp Res Rec. 2016;2583(1):99-109.

20. Savolainen PT, Mannering FL, Lord D, Quddus MA. The statistical analysis of highway crash-injury severities: a review and assessment of methodological alternatives. Accid Anal Prev [Internet]. 2011;43(5):1666-1676. Available from: https://doi.org/10.1016/j.aap.2011.03.025.

21. Iranitalab A, Khattak A. Comparison of four statistical and machine learning methods for crash severity prediction. Accid Anal Prev 2017;108(August):2736. Available from: https://doi.org/10.1016/j.aap.2017.08.008

22. Lord D, Mannering F. The statistical analysis of crash-frequency data: a review and assessment of methodological alternatives. Transp Res Part A [Internet]. 2010;44(5):291-305. Available from: https://doi.org/10.1016/j.tra. 2010.02.001

23. Cassarino M, Murphy G. Reducing young drivers' crash risk: are we there yet? An ecological systems-based review of the last decade of research. Transp Res Part F Traffic Psychol Behav [Internet]. 2018;56:54-73. Available from: https://doi.org/10.1016/j.trf.2018.04.003.

24. Connor J, Whitlock G, Norton R, Jackson R. The role of driver sleepiness in car crashes: a systematic review of epidemiological studies. Accid Anal Prev. 2001;33:31-41

25. Asbridge M, Desapriya E, Ogilvie R, Cartwright J, Mehrnoush V, Ishikawa T, et al. The impact of restricted driver's licenses on crash risk for older drivers: a systematic review. Transp Res Part A Policy Pract [Internet]. 2017:97:137145. Available from: https://doi.org/10.1016/j.tra.2017.01.006

26. Elvik R. Risk of road accident associated with the use of drugs: a systematic review and meta-analysis of evidence from epidemiological studies. Accid Anal Prev [Internet]. 2013;60:254-267. Available from: https://doi.org/10. 1016/j.aap.2012.06.017.

27. Oviedo-trespalacios $O$, Truelove $V$, Watson B, Hinton JA. The impact of road advertising signs on driver behaviour and implications for road safety: a critical systematic review. Transp Res Part A [Internet]. 2019;122(November 2018):85-98. Available from: https://doi.org/10.1016/j.tra.2019.01.012.

28. Moher D, Liberati A, Telzlaff J, Altman DG, Group P. Preferred reporting items for systematic reviews and meta-analyses: the PRISMA statement. PLoS Med. 2009;6(7).

29. World Health Orginization. Road traffic deaths, data by county [Internet]. Global Health Observatory data repository. 2019 [cited 2019 Oct 7]. Available from: https://apps.who.int/gho/data/node.main. A997.

30. Altman DG, Schulz KF. Statistical analyses and methods in the published literature: the SAMPL guidelines. In: Moher D, Altman DG, Schulz KF, Simera I, Wager E, editors. Guidelines for reporting health research: a user's manual. Oxford: John Wiley \& Sons, Ltd.; 2014. p. 265-74.

31. Lui K, Marchbanks P. A study of the time between previous traffic infractions and fatal automobile crashes, 1984-1986. J Saf Res. 1990:21:45-51.

32. Perneger T, Smith GS. The driver's role in fatal two-car crashes: a paired "case- control" study. Am J Epidemiol 1991;134(December):1138-1145.

33. Rajalin S. The connection between risky driving and involvement in fatal accidents. Accid Anal Prev. 1994;26(5):555-62.

34. Cooper PJ. The relationship between speeding behaviour (as measured by violation convictions) and crash involvement. J Saf Res. 1997:28(2):83-95.

35. Wundersitz $L$, Burns N. Relationships between prior driving record, driver culpability, and fatal crash involvement; 2004.

36. Kim HS, Kim HJ, Son B. Factors associated with automobile accidents and survival. Accid Anal Prev 2006;38(April):981-987.

37. Blower D, Green PE. Type of motor carrier and driver history in fatal bus crashes. Transp Res Rec J Transp Res Board. 2010:37-43.

38. Malchose D, Vachal K. Identifying factors that predict teen driver crashes [Internet]. 2011. Available from: http://www.mountain-plains.org/pubs/pdf/ MPC11-232.pdf

39. Lueck MD, Murray DC. Predicting truck crash involvement: linking driver behaviors to crash probability. J Transp Law, Logist Policy. 2011:109-28.
40. Gates J, Dubois S, Mullen N, Weaver B, Bedard M. The influence of stimulants on truck driver crash responsibility in fatal crashes. Forensic Sci Int. 2013;228:15-20.

41. Factor R. The effect of traffic tickets on road traffic crashes. Accid Anal Prev [Internet]. 2014;64:86-91. Available from: https://doi.org/10.1016/j.aap.2013. 11.010.

42. Reguly P, Dubois S, Bedard M. Examining the impact of opioid analgesics on crash responsibility in truck drivers involved in fatal crashes. Forensic Sci Int. 2014:234:154-61.

43. Dubois S, Mullen N, Weaver B, Bedard M. The combined effects of alcohol and cannabis on driving: impact on crash risk. Forensic Sci Int. 2015;248:94-100.

44. Kumfer W, Wei D, Liu H. Effects of demographic and driver factors on single-vehicle and multivehicle fatal crashes investigation with multinomial logistic regression. Transp Res Rec J Transp Res Board. 2015;2518:37-45.

45. Feng $\mathrm{S}, \mathrm{Li} Z \mathrm{Z}, \mathrm{Ci} Y$, Zhang G. Risk factors affecting fatal bus accident severity: their impact on different types of bus drivers. Accid Anal Prev [Internet]. 2016;86:29-39. Available from: https://doi.org/10.1016/j.aap.2015.09.025.

46. Li G, Chihuri S, Brady J. Role of alcohol and marijuana use in the initiation of fatal two-vehicle crashes. Ann Epidemiol [Internet]. 2017;27(5):342-347. Available from: https://doi.org/10.1016/j.annepidem.2017.05.003.

47. Hamzeie R, Thompson I, Roy S, Savolainen PT. State-level comparison of traffic fatality data in consideration of Marijuana Laws. Transp Res Rec J Transp Res Board [Internet. 2017;2660:78-85 Available from: https://doi.org/ 10.3141/2660-11.

48. Stringer RJ. Exploring traffic safety culture and drunk driving: an examination of the community and DUI related fatal crashes in. Transp Res Part F Psychol Behav [Internet]. 2018;56:371-80. Available from: https://doi. org/10.1016/j.trf.2018.05.014.

49. Mashhadi M, Wulff S, Ksaibati K. A comprehensive study of single and multiple truck crashes using violation and crash data. Open Transp J. 2018; $12 \cdot 43-56$

50. Yuan Y, Yang M, Gan Z, Wu J, Xu C, Lei D. Analysis of the risk factors affecting the size of fatal accidents involving trucks based on the structural equation model. Transp Res Rec. 2019;1-3.

51. Zhang X, Qu X, Tao D, Xue H. The association between sensation seeking and driving outcomes: a systematic review and meta-analysis. Accid Anal Prev [Internet]. 2019;123(127):222-34. Available from: https://doi.org/10. 1016/j.aap.2018.11.023

52. Tabachnick B, Fidell L. Using multivariate statistics. 5th Editio. Pearson Education, Inc.; 2007.

53. Yannis G, Richter T, Ruhl S, Dragomanovits A, Graham D, Laiou A, et al. Road traffic accident prediction modelling: a literature review. Transport. 2017; 170(TR5):245-54

\section{Publisher's Note}

Springer Nature remains neutral with regard to jurisdictional claims in published maps and institutional affiliations.

Ready to submit your research? Choose BMC and benefit from:

- fast, convenient online submission

- thorough peer review by experienced researchers in your field

- rapid publication on acceptance

- support for research data, including large and complex data types

- gold Open Access which fosters wider collaboration and increased citations

- maximum visibility for your research: over $100 \mathrm{M}$ website views per year

At BMC, research is always in progress.

Learn more biomedcentral.com/submissions 